\title{
Safety and immunogenicity of a randomized phase I prime-boost trial with ALVAC-HIV (vCP205) and gp160 MN/LAl-2 adjuvanted in alum or polyphosphazene
}

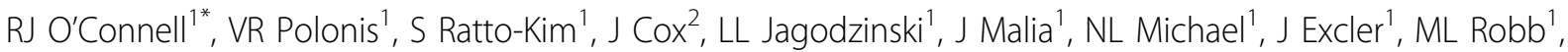 \\ $\mathrm{JH} \mathrm{Kim}{ }^{1}$
}

From AIDS Vaccine 2012

Boston, MA, USA. 9-12 September 2012

\section{Background}

ALVAC-HIV prime/HIV-1 Env protein boost regimens have shown HIV-specific neutralizing antibody (NAb) and cell-mediated immune responses, but the impact of protein subunit schedule and adjuvant requires further definition.

\section{Methods}

A Phase 1 trial was conducted in two parts. In Part A, (open-label) 19 volunteers received oligomeric gp160 MN/LAI-2 (ogp160) with a dose escalation (25, 50, 100 $\mu \mathrm{g})$. In Part B, 72 volunteers (60 active, 12 placebo) received placebo or recombinant canarypox expressing HIV-1 antigens, (ALVAC-HIV, vCP205) prime with different doses and schedules of ogp160MN/LAI-2 in alum or polyphosphazene (PCPP).

\section{Results}

The vaccines were safe and well tolerated with no vaccine-related serious adverse events. Cumulative chromium release CTL frequency was $37 \%$, and $54 \%$ of volunteers showed proliferative responses to HIV antigens. Lymphoproliferative CD4+, HIV-specific responses were seen in $53 \%$ of ogp 160 only and $57 \%$ of primeboost recipients, respectively. Induced binding antibody to ogp160 was dose-dependent. NAb responses to vaccine homologous Tier 1 HIV-1 MN were seen in $99 \%$ of vaccine recipients. While NAb to the heterologous Tier 2 US-1 (R5, clade B) pseudovirus was negative in all

${ }^{1}$ U.S.Military HIV Research Program, Bethesda, MD, USA

Full list of author information is available at the end of the article volunteers tested using TZM-bl cells, in a PBMC-based assay, US-1 primary isolate $\mathrm{Nab}$ was induced in $2 / 19$ (10.5\%) recipients of ogp160 protein alone and in 5/30 (16.7\%) prime-boost volunteers who received ogp160 in PCPP. Primary isolate neutralization was observed more frequently overall in recipients of ogp160 in PCPP, as compared with alum $(\mathrm{p}=0.027)$. Using an intracellular p24 flow-cytometry assay, sera from an ALVAC-HIV/ ogp160 recipient demonstrated $94 \%$ neutralization of US-1.

\section{Conclusion}

A small percentage of vaccine recipients developed $\mathrm{Nab}$ to heterologous primary isolates, responses that to our knowledge have not been previously described. These results constitute proof of concept that Tier 2 NAb can be elicited by vaccination in humans, and underscore the importance of further optimization of prime-boost vaccination and adjuvanting strategies for HIV-1 prevention.

\section{Author details}

'U.S.Military HIV Research Program, Bethesda, MD, USA. ${ }^{2}$ International AIDS Vaccine Initiative, New York, NY, USA.

Published: 13 September 2012

doi:10.1186/1742-4690-9-S2-050

Cite this article as: $O^{\prime}$ Connell et al: Safety and immunogenicity of a randomized phase I prime-boost trial with ALVAC-HIV (vCP205) and gp160 MN/LAl-2 adjuvanted in alum or polyphosphazene. Retrovirology 2012 9(Suppl 2):050. 